\title{
Design of an Inductive Contactless Power System for Multiple Users
}

\author{
Fredrik F. A. Van der Pijl, Jan. A. Ferreira, Pavol Bauer, Henk Polinder \\ Electrical Power Processing group of the Faculty of Electrical Engineering, Mathematics and Informatics \\ Technical University of Delft \\ Delft, The Netherlands \\ f.vanderpijl@ewi.tudelft.nl
}

\begin{abstract}
The technique of inductive contactless energy transfer (CET) has found its application in specific industrial applications where an (air) gap between energy source and load is preferable or even required. A relative new application area of CET is where CET is part of a general energy distribution network. As an example, electrical sockets might give problems in bathrooms for safety reasons: CET provides a solution because of inherent electrical isolation between source (grid) and loads (e.g. hair dryer or electrical shaver). For such application this paper reports on the design of a contactless power system that is able to supply to multiple users simultaneously, analogously to the regular plug-and-socket network. The proposed contactless system consists of a special energy supply cable where clamps (each maximum $240 \mathrm{~W}$ ) can be mounted on at arbitrary locations along the cable while electrical isolation is maintained between cable and clamps. Result is a supply cable design and a prototype with 2 clamps, which are compared by means of analytical calculations, simulations and experimental results.
\end{abstract}

Keywords-contactless; energy transfer; inductive coupling; multiple output; power transfer

\section{INTRODUCTION}

$\mathrm{T}$ $\mathrm{HE}$ technique of using an inductive magnetic coupling to transfer energy has already found its use in several specific industrial applications. For example, in the research field of precision positioning, energy supply cables which are attached to a moving object disturb the positioning process of the object [1]. In the latter example the contribution of contactless energy transfer, increasing the precision, is apparent. Precision positioning and other specific industrial applications benefit from contactless energy transfer as being the only alternative [2] [3] [4] [5].

This paper explores a different type of application, where contactless transfer is part of a general energy supply chain, rather than that it performs an integrated function within a specific electrical device.

Focus is on applications were energy supply is required to be geometrically flexible. Examples form exposition halls and music stadiums, where the position of (electrical) equipment differs per exposition and concert. In this paper a contactless system is proposed which inherently is flexible in use: electrical devices can be clamped on (by means of a universal clamp) at an arbitrary position along a special electrically isolated supply cable. Thereby we avoid the problem of the current plug-and-sockets, where a plug can only be connected to one of the (few) sockets.

Also, such electrically isolated supply cable can be applied in wet environments, in for example bathrooms or for the underwater-equipment of professional divers.

Objective for this paper is to introduce an energy supply cable, based on inductive energy transfer, which is accessible everywhere along the cable by multiple users. The prototype that has been build contains two clamps which output DC voltage and current for a purely resistive load (e.g. user). It is noted that a related application area from literature consists of contactless planar DC charging platforms for (mobile) electrical devices [6] [7].

Theory behind the $D C$ cable design is not new [8] [9], but results will be used for near-future work on an $A C$ multi-user cable design. Also, a (feedback) controller has not been implemented for the prototype, while focus is on steady-state system functioning at a large load-range. The design makes use of results from [10], [11] and [12].

Chapter II introduces the concept. Chapter III discusses the practical design and a model of the system. In chapter IV simulation methodology and implementation is explained in order to compare analytical and simulation results with experimental results in chapter V. Chapter VI concludes the work.

\section{CONCEPT DESCRIPTION}

The proposed contactless supply cable can be compared in functionality to a standard cable with several sockets. Difference between standard plug-and-socket system and the proposed contactless system concept in Fig. 1 is the respective use of plugs and clamps. Plugs are attached to sockets, while clamps are connected at arbitrary locations along the contactless cable. 


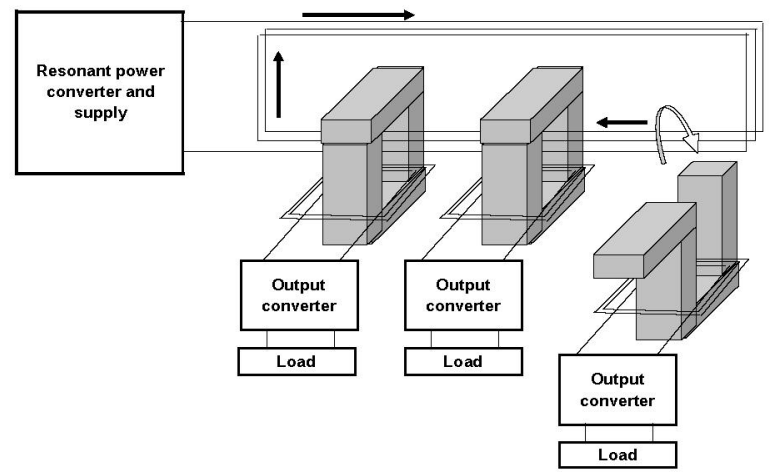

Fig. 1. Proposed contactless system with two clamps attached and one ready to be connected. Arrows next to the conductor spiral denote current direction

Each clamp-cable connection can be seen as a special transformer. To obtain a reasonable magnetic core size for the clamp-cable transformer the system operating frequency is required to be several orders of magnitude larger than $50 \mathrm{~Hz}$. Therefore, a frequency-converter is required between the utility grid and the proposed cable (e.g. input converter), which is abundant in the standard system. For the same reason, an output converter in between clamp and load is required.

\section{A. Clamp-cable transformer}

The contactless cable consists of a single (primary) conductor that is wound according to Fig. 2. Result is an inner-winding section and an outer-winding section where the current in both sections flows opposite. If the outer-section windings are distributed equally and tight across the outer periphery, zero-magnetic field outside the cable is obtained (by the canceling currents).

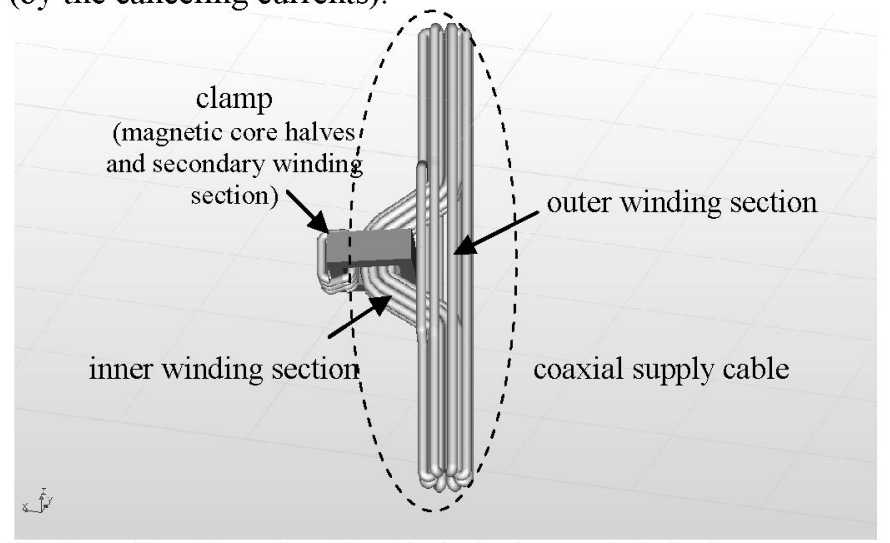

Fig. 2. Coaxial supply cable with single clamp as built for the prototype

To transfer energy from the cable to a clamp the magnetic cores of the clamp must encircle one of both winding sections. It was decided to pull-out the inner-winding section locally at which position a clamp is attached. This action can be compared to putting a plug in a socket. Each clamp is composed of two separatable magnetic core halves and a fixed (secondary) winding.

Fig. 3 shows that the standard cable is a parallel system (in electrical current sense), while the contactless cable is a series system. The input current is distributed across the loads in the standard system while being shared by all clamps in the contactless system.

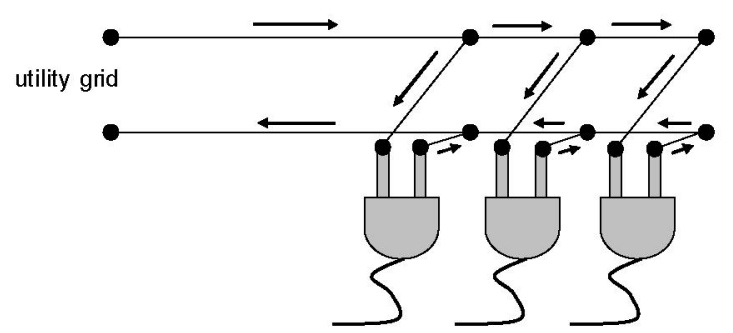

Fig. 3. Parallel plug-and-socket system in electrical current sense. Arrows denote current direction.

\section{B. Input and output converters}

The input converter in between utility grid and cable has two tasks. First task is to convert the utility's $50 \mathrm{~Hz}$ to a higher frequency to meet a reasonable magnetic core-size for the clamp. Second task is to regulate the power throughput from the grid to the variable number of clamps. In the prototype each output converter consists of a passive diode bridge rectifier with filter capacitor, to generate a DC output voltage across the resistive loads.

\section{Design requirements}

The proposed system is intended for domestic and office environments with a power range from $\mathrm{W}$ to $\mathrm{kW}$. The prototype considers two clamps of each $240 \mathrm{~W}$ maximum. Nominal output voltage is set at $240 \mathrm{~V}$, which is the rms-value of the $50 \mathrm{~Hz}$ utility grid voltage. Therefore, the output current ranges from (near) 0 to $1 \mathrm{~A}$.

All system components are rated at maximum power throughput. In practice, the average number of clamps that are attached at a time instant will be (much) less than the maximum allowed. Therefore, applicability of the proposed system depends on the wide-range efficiency. This paper explores maximum (practical) efficiency system conditions for the complete load range.

\section{SYSTEM DESIGN}

\section{A. Clamp-cable transformer}

Ferrite magnetic cores are chosen to maximize the inductive coupling factor of the clamp-transformer. A high coupling factor results in a large magnetizing inductance. A coreless design would avoid core loss, but this advantage will be small compared to the increase in copper loss. Fig 4 shows the situation where a single clamp is connected with a small part of the inner winding section of the cable in Fig. 2. Core material and dimensions as used in the prototype are given in Table I. 


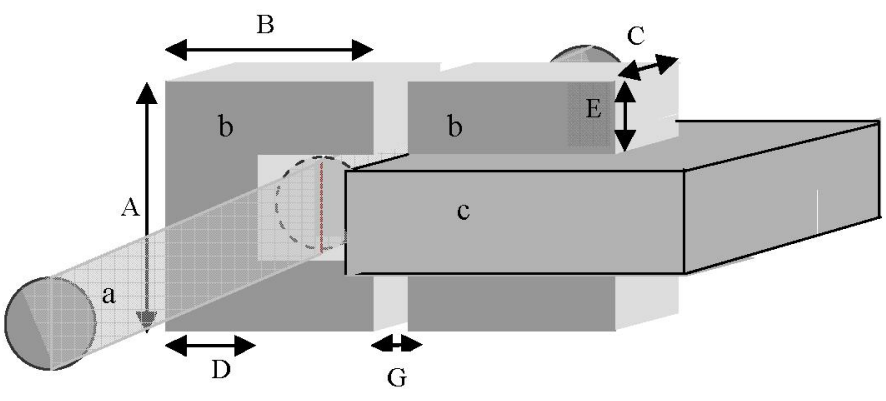

Fig. 4. Single clamp configuration as used in the prototype, with dimensions $\mathrm{A}-\mathrm{E}$ and a) inner winding section of cable, b) U-core, c) clamp winding section

TABLE I

PROTOTYPE TRANSFORMER DIMENSIONS FOR TWO-CLAMP CABLE AS REFERRED TO FIG. 4

\begin{tabular}{ccc}
\hline \hline \multirow{2}{*}{ Symbol } & Quantity & Value \\
\hline- & core material grade & $3 \mathrm{C} 80$ \\
- & core size & $\mathrm{U} 25 / 20 / 13$ \\
$\mathrm{~A}$ & & $46.5[\mathrm{~mm}]$ \\
$\mathrm{B}$ & & $14[\mathrm{~mm}]$ \\
$\mathrm{C}$ & See Fig. 4 & $3.16[\mathrm{~mm}]$ \\
$\mathrm{D}$ & & $3.25[\mathrm{~mm}]$ \\
$\mathrm{E}$ & & $30[\mathrm{~mm}]$ \\
$\mathrm{G}_{1}$ & air gap width of clamp 1 & $0[\mathrm{~mm}]$ \\
$\mathrm{G}_{2}$ & air gap width of clamp 2 & $0[\mathrm{~mm}]$ \\
\hline \hline
\end{tabular}

Objective in choosing a suitable core is to find an optimal balance between copper and iron loss to minimize their combined loss. From a thermal point of view the transformer must have sufficient surface to remove the loss by heat convection with the surrounding air.

The magnetic core size and material are determined according to the transformer design procedure in [12]:

1. Choose a material with the highest performance factor at the nominal operating frequency of the system

2. Minimize total (core and copper) loss for a range of available core sizes by tuning the core flux density, which guarantees optimal use of each individual core

3. Calculate maximum allowed transformer loss, which is given by the maximum heat removal capacity

4. Choose the minimum core size that does not exceed the maximum allowed loss

It is remarked that this procedure gives only an indication for a suitable core material and size. Further detailed design improvements as for example in [13] are not covered.

First step is to choose a material with high performance at the system operating frequency. The expression for iron loss is given in (1),

$$
\sum_{a=0}^{n_{c \text { clamps }}} P_{f e}=\sum_{a=0}^{n_{\text {clamps }}} K_{c} f^{\alpha} \widehat{B}^{\beta} V_{\text {core }}
$$

where $K_{c}, \alpha$ and $\beta$ are material specific constants that are empirically determined and given by the manufacturer. Their relative values determine a suitable frequency range for the material for a given power throughput. For example, from [5, p. 747] it is clear that ferrite grade $3 \mathrm{~F} 3$ is best for applications from 40 to $420 \mathrm{kHz}$. New core materials (lately the amorphous cores) are continuously under development with even higher (local) performance. $\widehat{B}$ is the peak flux density in the core and $V_{\text {core }}$ is the core volume. The sum $a$ is taken over the number of clamps $\mathrm{n}_{\text {clamps. }}$.

Copper loss is given in (2),

$$
\sum_{a=1}^{n_{\text {clamps }}} P_{C u, a}=\sum_{a=1}^{n_{\text {clamps }}} R_{C u, a} I_{a}^{2}=\sum_{a=1}^{n_{\text {ciamps }}} \frac{\rho \cdot n_{a}{ }^{2} \cdot l_{a}}{W_{C u}} I_{a}^{2}
$$

with copper resistance $\mathrm{R}_{\mathrm{Cu}}$ and rms-value of the current in the copper $\mathrm{I}_{\mathrm{a}}$. The sum $a$ is taken over the cable with subscript 0 and all secondary sides, which are equal to the number of clamps $\mathrm{n}_{\text {clamps. }}$. Resistance is subdivided in the specific copper resistance $\rho$, length of a single turn $1_{\mathrm{a}}$, number of turns $\mathrm{n}_{\mathrm{a}}$ and the total copper cross sectional area of all windings $\mathrm{W}_{\text {Cua }}$. $\mathrm{W}_{\mathrm{Cu}, \mathrm{a}}$ divided by $\mathrm{n}_{\mathrm{a}}$ is the cross sectional area of a single conductor.

Though optimum flux density in the core depends on the number of clamps, for clearness further design steps are performed for a single clamp. For multiple clamps the procedure is the same.

Second step is to determine the optimum flux density for a specific core size to obtain minimal loss at the required power throughput. Flux density, and thus optimum density, is controlled by varying the absolute number of windings. Starting from the law of Faraday, the number of primary windings $n_{0}$ is found to be related to peak value of the primary flux linkage $\lambda_{0}$, the peak core flux density $B$ and the core cross section $A_{c}$ (perpendicular to the direction of $B$ ) in (3),

$$
n_{0}=\frac{\hat{\lambda}_{0}}{2 A_{c} \widehat{B}}
$$

where $\lambda_{0}$ is assumed triangular, because of the block voltage across the magnetizing inductance as introduced in Fig. 7.

Substituting (3) in (2), minimal loss is found by setting the derivative of total loss to core flux density to zero, which implies equal but opposite derivates of copper and iron loss in (4).

$$
\frac{\delta P_{\text {tot }}}{\delta B}=0 \stackrel{\text { implies }}{\longrightarrow} \frac{\delta P_{C u}}{\delta B}=-\frac{\delta P_{F e}}{\delta B}
$$

Solving (4) gives the expression for the optimal peak flux density in (5). 


$$
\widehat{B}_{o p t}=\left(\frac{\rho 2 l_{c a b l e}}{W_{C u, 0}} \cdot \frac{I_{0}^{2}}{\beta K_{c} f^{\alpha} l_{e}} \cdot \frac{\hat{\lambda}_{0}^{2}(f)}{A_{c}^{3}}\right)^{\frac{1}{\beta+2}}
$$

Substituting this optimal density in the loss expressions (1)

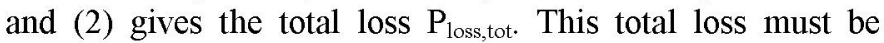
smaller than the heat removal capacity of the transformer as given in (6), where a uniform heat distribution inside the transformer is assumed.

$$
P_{\text {loss,tot, allowed }}=\frac{T_{s}-T_{a}}{R_{\theta}}=\left(T_{s}-T_{a}\right) \cdot \theta_{\text {heat }} A_{s}
$$

$T_{s}$ is the maximum allowed surface temperature of the core and $T_{a}$ is the maximum expected (and thus allowed) ambient temperature. The heat convection coefficient $\theta_{\text {heat }}$ for the transformer surface to air is assumed $7.5 \mathrm{~W} / \mathrm{m}^{2} \cdot \mathrm{K}$. $A_{s}$ is the total transformer-to-air boundary surface.

For each commercially available core $P_{\text {loss tot }}$ (which is optimal for that core size) and $P_{\text {loss,tot, allowed }}$ can be determined. The smallest (and thus cheapest) core that ensures $P_{\text {loss,tot }} \leq P_{\text {loss, tot, allowed }}$ is then selected. It must be noted that a larger core might result in less loss, but at larger expenses.

The above procedure for usual ferrite-core transformer design results in an optimum flux density between 0.1 and 0.2 $\mathrm{T}[11, \mathrm{p} .769]$, that is well below the usual core saturation flux density of around $0.4 \mathrm{~T}$. This interesting result is explained from the fact that iron loss becomes more dominant at relative high operating frequencies. Therefore, iron loss is traded for higher copper loss. Different from usual transformer design is the large amount of primary copper that is located in the contactless supply cable. Therefore, optimal balance between copper and iron loss in the clamp-cable case results in a higher flux density. Fig. 5 illustrates this principle. Two situations are plotted, with $\mathrm{B}_{\text {usual }}$ and $\mathrm{B}_{\text {cable }}$ respectively the optimal flux density for a usual transformer and the cable with clamps, with large copper loss.

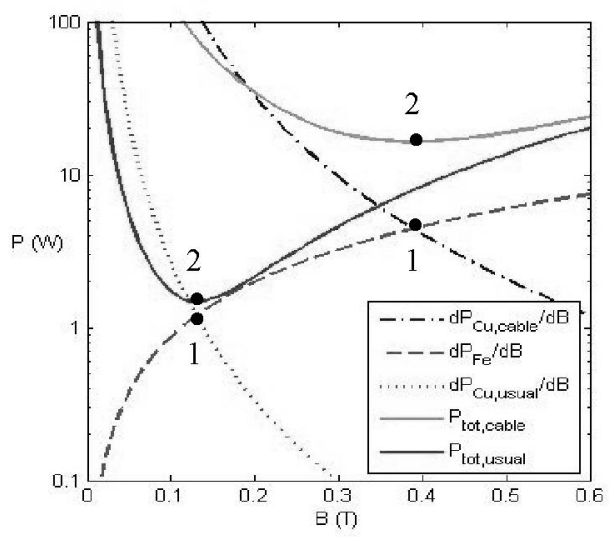

Fig. 5. Demonstration of a higher optimal flux density in case of larger copper loss and given core size. The intersection points of the individual loss derivatives at 1 coincide with the flux density at which the total losses reach their minima at 2 .

The procedure can be repeated for a range of frequencies, because the system operating frequency is a design choice. In general it will turn out that a higher frequency results in lower transformer loss, but this loss reduction is counteracted by the switching loss that increases with higher frequency. For the prototype design the latter trade-off has been omitted and an operating frequency around $100 \mathrm{kHz}$ was assumed design target. To conclude, Table II lists parameter values for the prototype as used in this section.

TABLE I

PARAMETER VALUES FOR TWO-CLAMP CABLE PROTOTYPE AS REFERRED TO SECTION III-A

\begin{tabular}{ccc}
\hline \hline Symbol & Quantity & Value \\
\hline$\rho$ & specific copper resistance & $1.724 \cdot 10^{-8}[\Omega \mathrm{m}]$ \\
$l_{\text {cable }}$ & length of the supply cable & $2[\mathrm{~m}]$ \\
$\hat{\lambda}_{0}$ & peak value of flux linkage & $2.46 \cdot 10^{-4}[\mathrm{~V} / \mathrm{Hz}]$ \\
$\alpha$ & core loss specific constant & 1.42 \\
$\beta$ & switching frequency & 2.2 \\
$K_{c}$ & core cross sectional area & 2.06 \\
$f$ & total copper cross section in & $81[\mathrm{kHz}]$ \\
$A_{c}$ & transformer window & $33\left[\mathrm{~mm}^{2}\right]$ \\
$W_{C u, 0}$ & supply cable cross sectional area & $100\left[\mathrm{~mm}^{2}\right]$ \\
$A_{s}$ & number of windings in the cable & $0.065\left[\mathrm{~m}^{2}\right]$ \\
$n_{0}$ & number of clamps & 7 \\
$n_{c l a m p s}$ & & 2 \\
\hline \hline
\end{tabular}

\section{B. Supply cable and input converter}

Two reasons indicate the use of resonant circuit operation. First reason is that magnetic field inside the cable at positions where there is no clamp attached is considered as leakage inductance. Despite a small distance between inner and outer sections of the cable, a large voltage drop occurs across the latter relative large inductance. This voltage drop can be compensated by a capacitor. Second reason is to reduce switching loss at the relative high switching frequency of 100 
$\mathrm{kHz}$ by zero-current and/or zero-voltage switching. To obtain a series resonant circuit, a capacitor $\mathrm{C}_{\text {res }}$ is placed in series with the cable and connected with switches in full-bridge configuration, as shown in Fig. 4. The resonant capacitor is chosen to obtain a resonant frequency of near $100 \mathrm{kHz}$ with the given $L_{\sigma 0}$ as introduced in (7). For the prototype, the utility grid with rectifier is represented by a DC voltage supply.

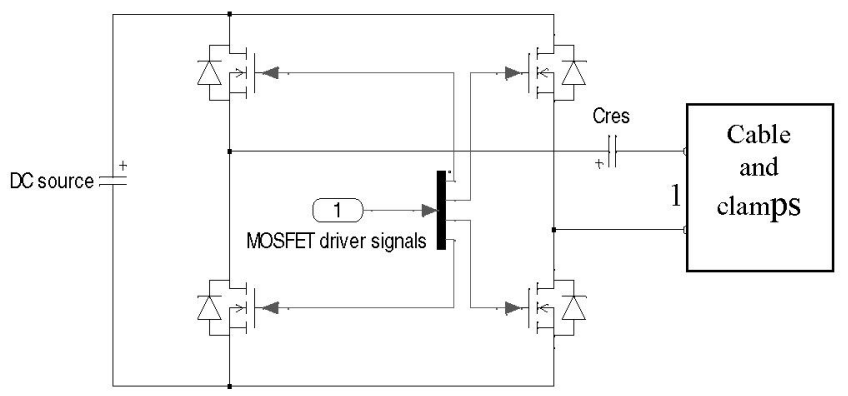

Fig. 6. MOSFET full-bridge and resonant capacitor $\left(\mathrm{C}_{\mathrm{res}}\right)$ as DC-AC input converter. The utility grid and the following rectifier are represented by a DC voltage source. Position 1 refers to the same position in Fig. 7.

The leakage inductance of the supply cable is dependent on the number of clamps that are attached at that time instant. The primary leakage inductance is denoted by $L_{\sigma 0}$ and calculated as in (7).

$$
\begin{aligned}
& L_{\sigma 0}=\frac{\mu_{0} \cdot n_{0}^{2} \cdot A_{\text {cable }}}{l_{m, a v}} \\
& A_{\text {cable }}=\left(l_{\text {cable }}-n_{\text {clamps }} C\right) \cdot\left(r_{2}-r_{1}\right) \\
& \frac{1}{l_{m, a v}}=\frac{1}{r_{2}-r_{1}} \int_{r_{1}}^{r_{2}} \frac{1}{l_{m}(r)} d r=\frac{\ln \left(r_{2}\right)-\ln \left(r_{1}\right)}{2 \pi \cdot\left(r_{2}-r_{1}\right)}
\end{aligned}
$$

where $A_{\text {cable }}$ is the surface area inside the cable perpendicular to the direction of the flux, $l_{m, a v}$ the average length of the magnetic flux path and $r_{1}$ and $r_{2}$ respectively are the inner and outer winding radii.

\section{Power transfer model}

System translation into the electrical domain is useful for exploring full system behavior including input and output converters. With two clamps attached to the cable the equivalent electrical circuit is given in Fig. 7.

The primary leakage inductance $L_{\sigma 0}$ depends on the number of clamps by (7). This dependence is assumed relative small $\left(\mathrm{C}<1_{\text {cable }}\right)$. The resulting change in resonant frequency is to be acted upon by the controller of the input converter, which will be subject of a future paper.

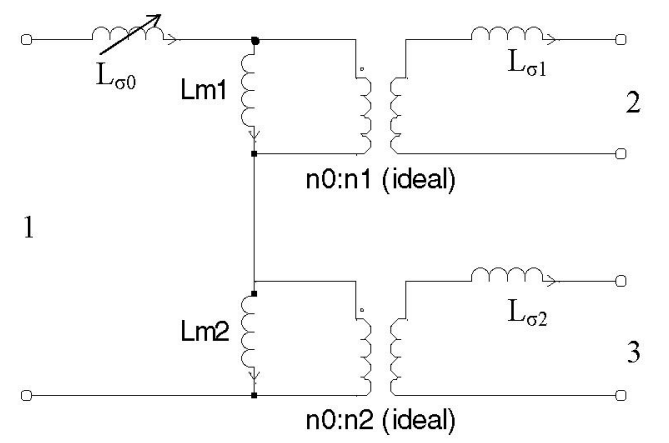

Fig. 7. Two-clamp cable equivalent electrical circuit. Position 1 refers to the same position in Fig. 6 and positions 2 and 3 refer to the same position in Fig. 8.

Magnetizing inductances $\mathrm{L}_{\mathrm{M} 1}$ and $\mathrm{L}_{\mathrm{M} 2}$ are found in (8) by assuming an effective magnetic path length $l_{e}$. Secondary side leakage inductance $L_{\sigma 1}$ and $L_{\sigma 2}$ are approximated with (9) from [11, p.780],

$$
\begin{aligned}
& L_{M 1}=L_{M 2}=\frac{\mu_{0} \mu_{r} \cdot n_{0}^{2} \cdot A_{c}}{l_{e}} \\
& L_{\sigma 1}=L_{\sigma 2}=\frac{\mu_{0} \cdot n_{[1,2]}^{2} \cdot l_{[1,2]}}{p_{[1,2]}^{2} \cdot h_{w}} \cdot\left(\frac{b_{C u}}{3}+b_{i}\right)
\end{aligned}
$$

where $p$ is the number of secondary winding layers, $h_{w}$ is the height of the winding window, $b_{C u}$ is the width of the copper, $b_{i}$ is the interwinding insulation thickness and $l$ is the length of a single turn. Brackets denote clamp 1 or clamp 2, which are assumed equal. Table II shows prototype

\begin{tabular}{|c|c|c|c|}
\hline Symbol & Quantity & $\begin{array}{l}\text { Measured } \\
\text { inductance }\end{array}$ & $\begin{array}{l}\text { Calculated } \\
\text { inductance }\end{array}$ \\
\hline$L_{m l, c a l c}$ & $\begin{array}{l}\text { calculated magnetizing } \\
\text { inductance clamp } 1\end{array}$ & $35.0[\mu H]$ & $39.2[\mu H]$ \\
\hline$L_{m 2, c a l c}$ & $\begin{array}{l}\text { calculated magnetizing } \\
\text { inductance clamp } 2\end{array}$ & $38.6[\mu H]$ & $39.2[\mu H]$ \\
\hline$L_{\sigma 0, \text { calc }}$ & $\begin{array}{l}\text { calculated leakage } \\
\text { inductance cable }\end{array}$ & $35.3[\mu H]$ & $32.7[\mu H]$ \\
\hline$L_{\sigma 1, \text { calc }}$ & $\begin{array}{c}\text { calculated secondary } \\
\text { leakage inductance } \\
\text { clamp } 1\end{array}$ & $49.0[\mu H]$ & $30.9[\mu H]$ \\
\hline$L_{\sigma 2, \text { calc }}$ & $\begin{array}{l}\text { calculated secondary } \\
\text { leakage inductance } \\
\text { clamp } 2\end{array}$ & $23.8[\mu H]$ & $30.9[\mu H]$ \\
\hline
\end{tabular}
measurements versus calculated inductances.

TABLE II

CALCULATED INDUCTANCES FOR TWO-CLAMP CABLE AS REFERRED TO EQUATIONS 10 AND 11

An output converter as in Fig. 8 is connected to locations 2 and 3 in Fig. 7. The output filter capacitor in Fig. 6 is chosen large enough to reduce the voltage (and current) ripple of the 
output.

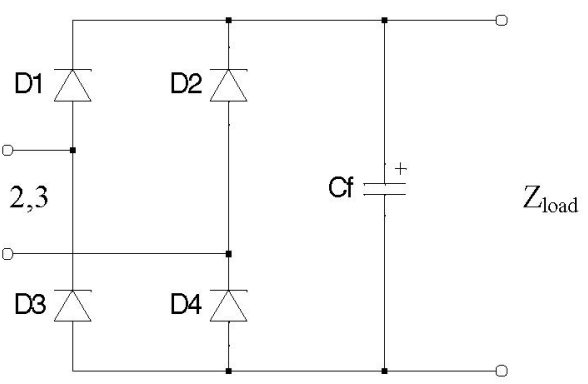

Fig. 8. Diode bridge rectifier and filter capacitor as AC-DC output converter

The LLC-type resonant converter topology with output rectifier, as given by the combination of Figs. 6, 7, and 8, is a well-studied topology. An analytical model can be found in [10], where in the analysis the output stage is represented by a voltage source that is put in parallel to the magnetizing inductance of the transformer. The voltage source changes polarity when the current through the diodes crosses zero. With $N$ clamps there are $N$ output voltage sources, but by assuming the clamps to be identical they can be combined into a single source, because the current though the diodes reaches zero at the same time instant for all clamps, and the analytical model in $[10]$ is applicable. When input, required output voltage, switching frequency and the LLC values are known, steady-state voltage and current waveforms at all system nodes can be calculated analytically. Following the model in [10], typical waveforms for the clamp-cable system are presented in Fig. 10.

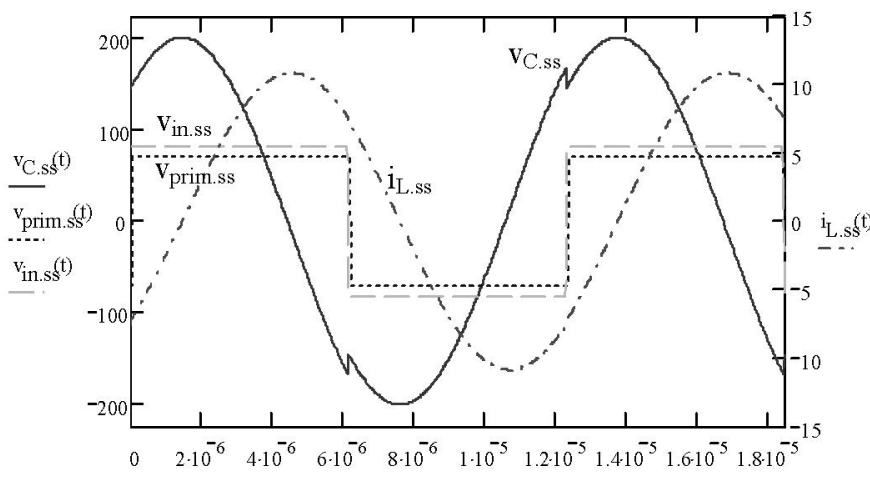

Fig. 9. Steady-state analytical waveforms of DC-DC cable for parameters as in Table II, with $\mathrm{i}_{\mathrm{Lss}}$ the resonant tank current, $\mathrm{v}_{\text {C.ss }}$ the voltage across the resonant capacitor, $v_{\text {inss }}$ the block-voltage across the input converter bridge, and $v_{\text {prim.ss }}$ the sum of the voltages across the magnetizing inductances of the two clamps.

\section{System loss}

System loss is subdivided into conduction loss of the input converter switches, copper loss in the supply cable, iron loss in the magnetic cores and conduction loss in the output diodes. Copper loss in the secondary windings is neglected in (13) as motivated by (10) and (11), where (10) assumes ideal coupling.

$$
\begin{aligned}
& n_{0}^{2} \hat{I}_{0}^{2}=n_{[1,2]}^{2} \hat{I}_{[1,2]}^{2}, \quad 2 l_{\text {cable }} \gg l_{[1,2]} \stackrel{\text { implies }}{\longrightarrow} \\
& P_{C u 0}=\frac{\rho \cdot n_{0}^{2} \cdot 2 l_{\text {cable }}}{W_{C u, 0}} \hat{I}_{0}^{2} \gg P_{C u[1,2]}=\frac{\rho \cdot n_{[1,2]}^{2} \cdot l_{[1,2]}}{W_{C u,[1,2]}} \hat{I}_{[1,2]}^{2}
\end{aligned}
$$

Also, switching loss is neglected in (14) because aboveresonance or resonant operation is assumed, which (ideally) implies zero-voltage and zero-current turn-on and losslesssnubber turn-off. Together with (1) and (2) the system loss model is complemented with switches conduction loss in (11) and diodes conduction loss in (12),

$$
\begin{aligned}
& P_{\text {sw, condloss,tot }}=n_{\text {switches }} \cdot v_{s w} \cdot \hat{I}_{0} \\
& P_{\text {diode, loss,tot }}=n_{\text {diodes }} v_{\text {diode }} \cdot \hat{I}_{[1,2]}=n_{\text {diodes }} v_{\text {diode }} \frac{\hat{I}_{0}}{n} \\
& P_{C u[1,2]} \stackrel{\text { assumed }}{\longrightarrow} 0 \\
& P_{\text {sw, swloss }} \stackrel{\text { assumed }}{\longrightarrow} 0
\end{aligned}
$$

where $n_{\text {switches }}$ and $n_{\text {diodes }}$ are the number of switches and diodes respectively that are conducting at the same time instant, $v_{s w}$ is the voltage drop across a conducting switch, $v_{\text {ctiode }}$ is the conduction resistance of a diode and $n$ is the transformer winding ratio.

TABLE III

PARAMETER VALUES FOR TWO-CLAMP PROTOTYPE AS REFERRED TO SECTIONS III-B, III-C AND III-D

\begin{tabular}{ccc}
\hline \hline Symbol & Quantity & Value \\
\hline$n$ & primary-secondary winding ratio & $6[-]$ \\
$r_{1}$ & radius of inner winding section & $7.5[\mathrm{~mm}]$ \\
$r_{2}$ & radius of outer winding section & (from the centre of the cable) \\
$b_{i}$ & interwinding insulation thickness & $40[\mathrm{~mm}]$ \\
$n_{1}, n_{2}$ & number of secondary windings for & $\sim 0[\mathrm{~mm}]$ \\
$n_{\text {switches }}$ & clamp 1 and 2 & $42[-]$ \\
$n_{\text {diodes }}$ & number of switches & $4[-]$ \\
$v_{\text {diode }}$ & number of diodes & $8[-]$ \\
$v_{s w}$ & forward voltage across diode & $1.05[\mathrm{~V}]$ \\
$l_{\text {cable }}$ & lorward voltage across switch & $1.4[\mathrm{~V}]$ \\
$b_{C u}$ & width of total copper cross section & in winding window \\
$p_{1}, p_{2}$ & number of secondary winding & $30[\mathrm{~mm}]$ \\
$h_{w}$ & layers for clamp 1 and 2 & $3[-]$ \\
$\mu_{0}$ & height of the winding window & $3[\mathrm{~mm}]$ \\
$\mu_{r}$ & magnetic permeability in vacuum & $4 \pi \cdot 10^{-7}[\mathrm{H} / \mathrm{m}]$ \\
\hline \hline
\end{tabular}


IV. SysteM SIMULATION OF DC-DC CABLE

The transformer core has been chosen large enough for a $240 \mathrm{~W}$ throughput at $240 \mathrm{~V}$. For a future $50 \mathrm{~Hz} \mathrm{AC}$ system, the output voltage will vary. Also, the required load power per clamp will vary from near-zero to $240 \mathrm{~W}$. Therefore, objective of the simulations is to quantify the system efficiency and component ratings at various load resistances and output voltages.

The analytical model from section III-C and [10] is informative when the output voltage is known exactly. A difficulty arises when the output voltage is not known exactly and the switching frequency is near resonance. In this situation a slight error in output voltage causes a large change in resonant current amplitude because only voltages are specified in the model. In practice this will not occur, because the resistive load will damp the system, which is clear from the voltage-current relation of a resistor.

To overcome the difficulty, the system topology has been simulated to find power throughput (with variable load resistances) versus efficiency. The topology was simulated lossless to find the peak resonant current, which was then inserted in (1), (2), (11) and (12) to calculate system loss. The resulting loss can be added to the simulated model as resistances and simulated again to converge to more precision. Simulated power throughput versus efficiency is compared with measurements in section V.

\section{COMPARISON BETWEEN MEASUREMENTS AND MODEL}

Objective of this section is to relate simulation results with measurements from the prototype as presented in Fig. 10. Implementation details are shown in Fig. 11. Component values as used for the prototype are listed in previous tables. The prototype was build with already available materials in the laboratory. Therefore, non-optimal components were implemented. Especially, core material and size are far from optimal unfortunately. Typical waveforms measured at full load are presented in Fig. 12, where the almost perfect sinusoidal waveforms show that switching happens at the resonant frequency.

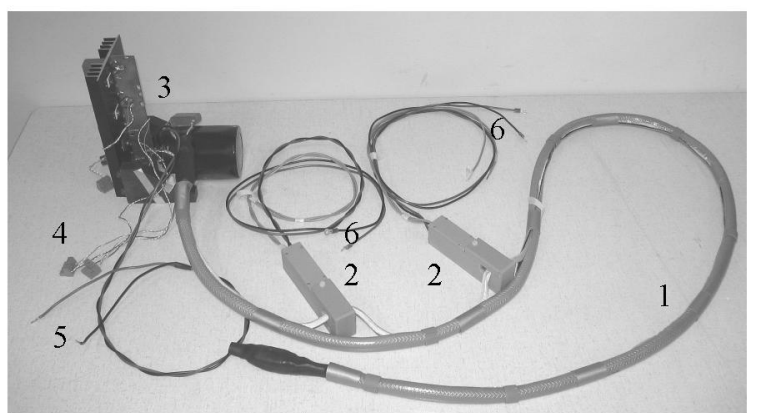

Fig. 10. Prototype contactless system, with 1) cable, 2) clamp, 3) input converter, 4) connectors for MOSFET driver circuitry, 5) connectors for DC voltage supply and 6) connectors for load

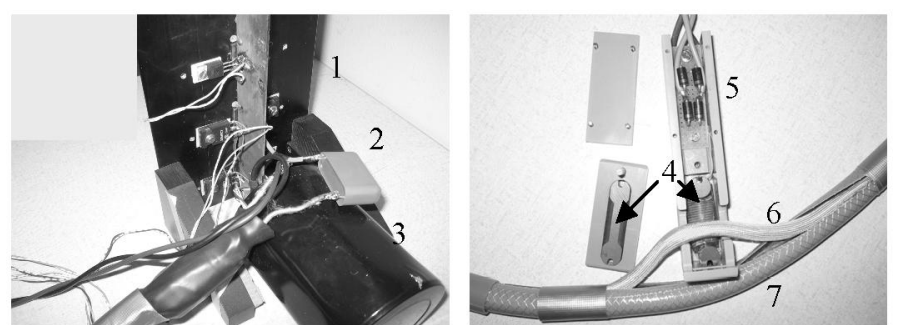

Fig. 11. Details of prototype system, with 1) MOSFET full-bridge with heat sink, 2) resonant capacitor $\mathrm{C}_{\text {res, }}, 3$ ) filter capacitor between DC voltage supply and MOSFET bridge to compensate for reactive power, 4) magnetic core half, 5) diode full-bridge, 6) pulled-out inner winding section and 7) outer winding section remains within the cable

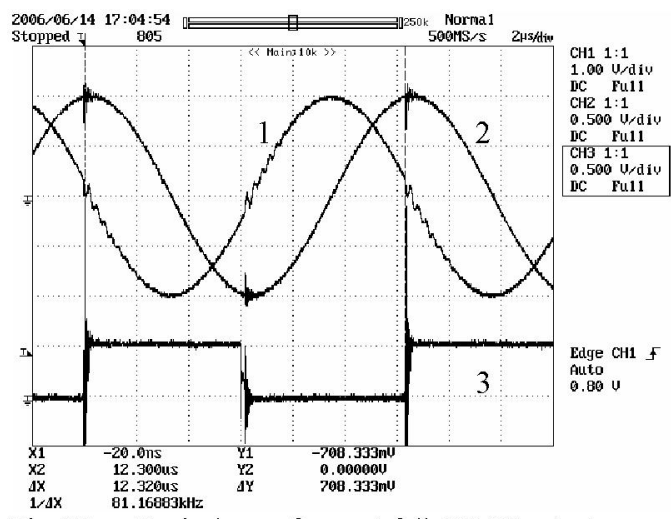

Fig. 12. Typical waveforms at full $480 \mathrm{~W}$ output power observed from the prototype system for a comparison with the analytical waveforms in Fig. 9. The resonant current on channel 1 at $1 \mathrm{~V} / \mathrm{div}$ is measured with a $0.1 \mathrm{~V} / \mathrm{A}$ probe. The voltage across the resonant capacitor on channel 2 at $0.5 \mathrm{~V} / \mathrm{div}$ is measured with a $200 \mathrm{x}$ attenuation factor. Channel 3 shows a driving signal for one phase-arm of the input converter bridge.

Measurement results are compared with simulation and calculation results in Figs. 13-15. For Figs. 13 and 14 two clamps were connected to the cable, while for Fig. 15 only a single clamp was used in order to reduce the power throughput of the system.

First aspect to notice is the relative large contribution of the core loss to the total loss, which was expected from the inappropriate $3 \mathrm{C} 80$ core material and size. This loss can be reduced substantially with the implementation of a proper core. Furthermore, efficiency is relative flat in the complete power range covered by Figs. 13-15. Fig. 13 shows a total output power of $480 \mathrm{~W}$ decreasing to $280 \mathrm{~W}$ with an almost constant efficiency. For Fig. 14 the input voltage is decreased from $80 \mathrm{~V}$ to $10 \mathrm{~V}$, where the output power decreases from $400 \mathrm{~W}$ to less than $7 \mathrm{~W}$. Also in this case the measured efficiency curve is rather flat. It must be noted that the (extremely high) measured efficiencies for small input voltage lack precision, because of analog meter reading errors. Fig. 15 shows results for a single clamp with variable load resistance connected. Efficiency for the rated power of $240 \mathrm{~W}$ is little less than for two clamps, because primary loss in this case is not shared across multiple clamps.

Perhaps most interesting is the fact that with suboptimal components an average efficiency of near $90 \%$ was established, which shows feasibility of the concept in practice. 


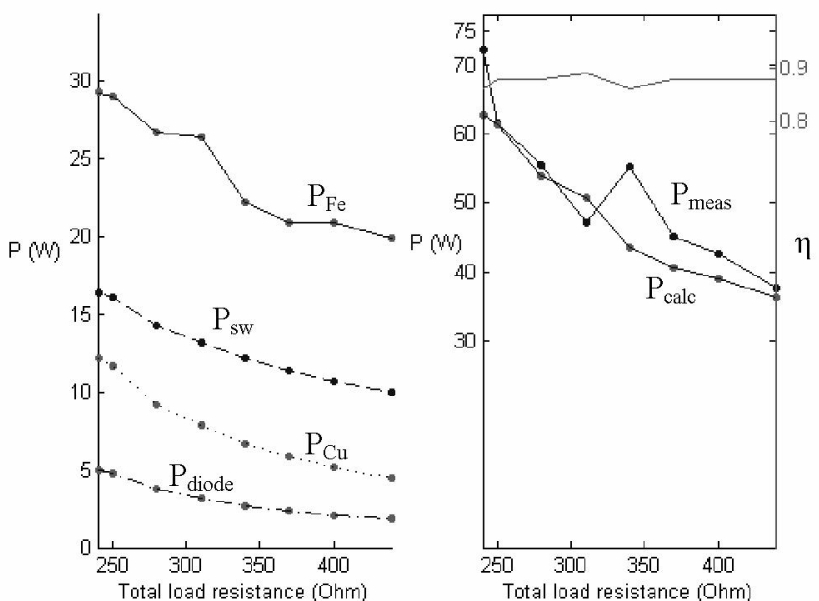

Fig. 13. Calculated and measured system loss and efficiency as a function of combined output resistance of two clamps. The left figure shows calculated iron, copper, conduction switching and diode loss. The right figure relates measured and calculated total loss. Measured system efficiency is shown on the right.
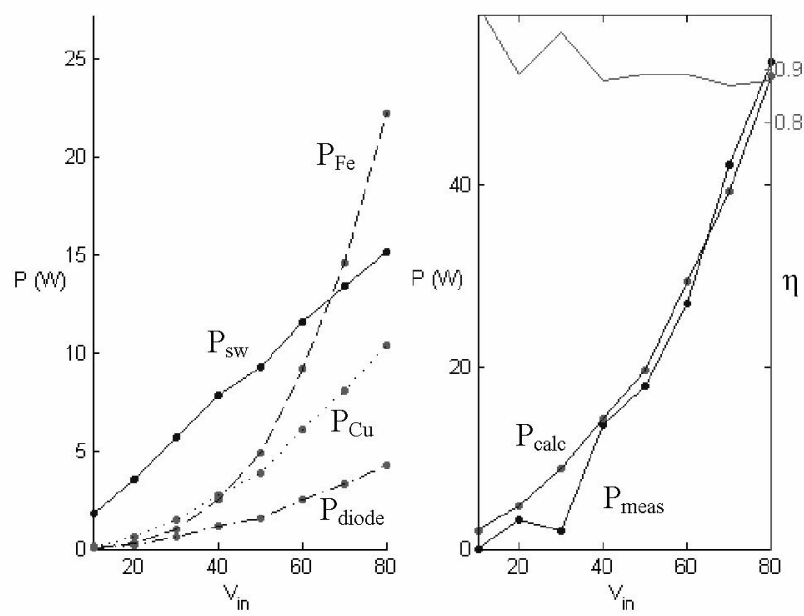

Fig. 14. Calculated and measured system loss and efficiency as a function of the system input voltage. The left figure shows calculated iron, copper, conduction switching and diode loss. The right figure relates measured and calculated total loss. Measured system efficiency is shown on the right.
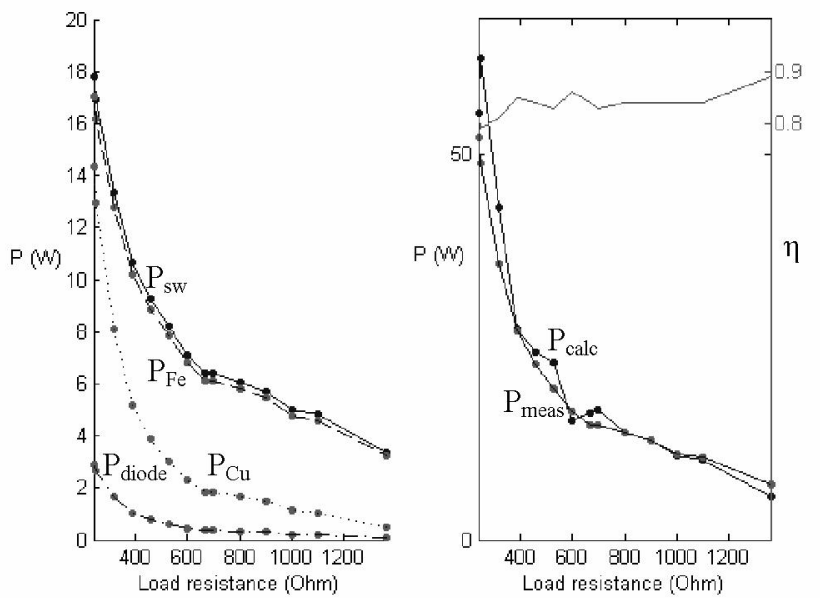

Fig. 15. Calculated and measured system loss and efficiency as a function of output resistance of a single clamp. The left figure shows calculated iron, copper, conduction switching and diode loss. The right figure relates measured and calculated total loss. Measured system efficiency is shown on the right.

\section{CONCLUSION}

A DC-DC system for transferring energy with electrical isolation between primary and secondary has been introduced and designed. Practical results from a prototype are backed-up by simulation results. Measured efficiencies of almost $90 \%$ with non-optimal system components in a large load range are promising. Future work comprises extension of the concept to an AC-AC multi-user system.

\section{REFERENCES}

[1] A. V. Lebedev, E. A. Lomonova, P. G. Van Leuven, J. Steinberg, D. A. H. Laro, "Analysis and initial synthesis of a novel linear actuator with active magnetic suspension", Conference Record of the $39^{\text {th }}$ Industry Applications Conference Annual Meeting, Vol. 3, 3-7 Oct. 2004, pp. 2111-2118

[2] K. W. Klontz, D. W. Novotny, "Contactless power delivery system for mining applications", IEEE Transactions on Industry Applications, Vol. 31, No. 1, January/February 1995

[3] A. W. Kelley, W. R. Owens, "Connectorless power supply for an aircraft-passenger entertainment system", IEEE Transactions on Power Electronics, Vol. 4, Issue 3, July 1989, pp. 348-354

[4] H. Matsuki, T. Matsuzaki, "Simulations of temperature rise on transcutaneous energy transmission by non-contact energy transmitting coils", IEEE Transactions on Magnetics, Vol. 29, No. 6, November 1993

[5] J. M. Kim, D. H. Kang, S. J. Jung, D. J. Bang, "Design of the transverse flux linear motor with the integrated contactless power supply", unpublished.

[6] S. Y. R. Hui, W. W. C. Ho, "A new generation of universal contactless battery charging platform for portable consumer electronic equipment", IEEE Transactions on Power Electronies, Vol. 20, Issue 3, May 2005, pp. $620-627$

[7] C. L. W. Sonntag, E. A. Lomonova, J. L. Duarte, A. J. A. Vandenput and A. J. M. Pemen, "Contactless Energy Transfer for Office and Domestic Applications", unpublished

[8] S. Valtchev, B. V. Borges, K. Brandisky and J. B. Klaassens, "Efficient Resonant Inductive Coupling Energy Transfer Using New Magnetic and Design Criteria," $36^{\text {th }}$ IEEE Conference on Power Electronics Specialists, pp. 1293-1298, June 12, 2005

[9] J. M. Barnard, J. A. Ferreira, J. D. van Wyk, "Sliding transformers for linear contactless power delivery", IEEE Transactions on Industrial Electronics, Vol. 44, Issue 6, December 1997, pp. 774-779

[10] J. F. Lazar, R. Martinelli, "Steady-state analysis of the LLC series resonant converter", $16^{\text {th }}$ Annual IEEE Applied Power Electronics Conference and Exposition, Vol. 2, March 4-8, 2001, pp. 728-735

[11] N. Mohan, T. M. Undeland, W. P. Robbins, Power Electronics: Converters, Applications and Design, $3^{\text {rd }}$ ed., New York: Wiley, 2003

[12] R. W. Erickson, Fundamentals of Power Electronics, $1^{\text {st }}$ ed., New York: Chapman and Hall, May 1997

[13] C. R. Sullivan, "Optimal choice for number of strands in a litz-wire transformer winding", IEEE Transactions on Power Electronics, Vol. 14, No. 2, March 1999 